\title{
On a New Source of Electromagnetic Radiation: Experimental Evidence
}

\author{
Vladimir A. Leus ${ }^{1} \&$ Barry Smith ${ }^{2}$ \\ ${ }^{1}$ Department of Electrical Engineering and Electronics, University of Liverpool, Liverpool, UK \\ ${ }^{2}$ Q-Technologies, Liverpool, UK \\ Correspondence: Vladimir A. Leus, Department of Electrical Engineering and Electronics, University of \\ Liverpool, Liverpool L69 3GJ, UK. E-mail: vladalex@liv.ac.uk
}

Received: March 18, 2014 Accepted: April 14, 2014 Online Published: May 16, 2014

doi:10.5539/apr.v6n3p127 URL: http://dx.doi.org/10.5539/apr.v6n3p127

\begin{abstract}
Electromagnetism deals with three very different types of fields: "static", "kinematical", and "dynamical". Fields of the first type originate in a stationary body - charged or magnetized. Fields of the second type arise from a uniform movement of a body - also charged or magnetized. Fields of the third type arise due to the accelerated movement of a charge, for instance, in an oscillatory LC-circuit. In the present paper we offer an experimental proof for existence in nature of a dynamical source which comes entirely from another mechanism. The fundamentals of this mechanism lie in the accelerated movement of a system of elementary currents, which are responsible for the appearance of macroscopic magnetization. This finding gives rise to a new method of studying magnetic materials and their structure.
\end{abstract}

Keywords: kinematic and dynamic types of induction, Faraday's law, pulse propagation, magnetic shielding, field-structure interaction

\section{Introduction}

The existence in nature of electro-kinematical fields has been proven experimentally by Rowland and Eihenwald more than century ago (Rowland, 1878; Eihenwald, 1956). The existence of magneto-kinematical fields has been proven experimentally during several recent decades (Zajev \& Dokuchajev, 1964; Kelly, 2004; Leus \& Zatolokin, 2006; Leus \& Taylor, 2011; Taylor \& Leus, 2012). At last, a theoretical substantiation was done in Leus (2013), whereby the principal difference between "kinematical" and "dynamical" fields is strictly proven on the basis of the wave equation. It became apparent that kinematical fields do not conform to the wave equation with a speed-factor $\mathbf{v}$ neither in classical nor in relativistic cases. When a charged body moves with a constant velocity $\mathbf{v}$ relative to the laboratory's proper coordinate system, its electric field $\mathbf{E}$ also moves with the same velocity. The corresponding magnetic field $\mathbf{B}=\frac{1}{c^{2}}(\mathbf{v} \times \mathbf{E})$ of the electro-kinematical origin (SI units) is present in the laboratory (Rowland-Eihenwald effect). When a magnetized body moves, its permanent magnetic field $\mathbf{B}$ also moves and corresponding electric field $\mathbf{E}=\mathbf{B} \times \mathbf{v}$ of the magneto-kinematical origin also exists in the laboratory (Zajev-Dokuchajev effect or Z-D effect in brief, 1964).

This is just the Z-D effect that was used in our experiments. As a permanent magnet travels with a constant velocity $\mathbf{v}$ past conducting wires, it induces electro-motive force $(\mathrm{em} f)$ inside them. Electromagnetic waves impinging an antenna also excite variable emf within it. Some bewildering special cases might occur. For example, an iron bar having sinusoidal magnetization, as it is shown in Figure 1, when moving across a straight wire induces in it an oscillatory emf quite similar to that excited by an impinging radio-wave. Furthermore, the commonly known "flux rule", which calculates emf (generated in a closed circuit) directly due to a changing magnetic flux, is a very useful tool for solving many practical problems. However, the "flux rule" is an integration procedure which summarises all details of the induction process, thereby indiscriminately combining two quite distinct types of electromagnetic induction: the motional-type (Z-D effect) and the transformer-type (Faraday law). All experiments, described below, are dedicated to discern between them. The problem of differentiating between the two types of induction was solved in our experimentation due to a convenient "yardstick"- a magnetic dipole. As a consequence, the objective reality of a new source of electromagnetic radiation has been proven. 


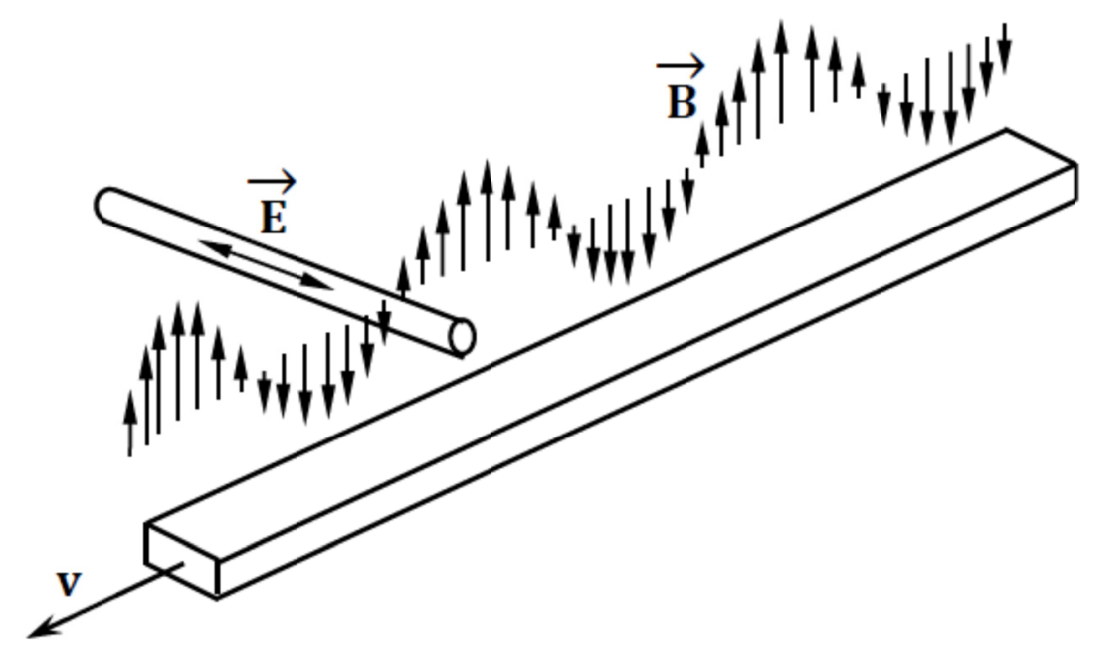

Figure 1. Kinematical interaction between a moving magnet and a crossing wire

\section{Experimental Equipment}

A sphere of diameter $d$ with a uniform magnetization $\mathbf{M}$ of magnitude $M$ and parallel to the $z$ axis is embedded in air (Figure 2). This sphere provides a magnetic field at each arbitrary point $A$ of the surrounding space. In total it is equal to the field of a dipole with the magnetic moment $\mathbf{p}_{m}=\frac{\pi d^{3}}{6} \mathbf{M}$. Let $(r, \theta, \varphi)$ be the system of spherical coordinates with polar axis directed along the vector $\mathbf{M}$ and with origin at the centre of the sphere. The field of magnetic dipole may be expressed through simple elementary functions (Jackson, 1998, p. 183). In this case the components of the magnetic flux density are

$$
B_{r}=p \frac{\cos \theta}{r^{3}}, B_{\theta}=\frac{p}{2} \frac{\sin \theta}{r^{3}}, B_{\varphi}=0,
$$

where $p$ is a constant numerical coefficient depending on the system of units used.

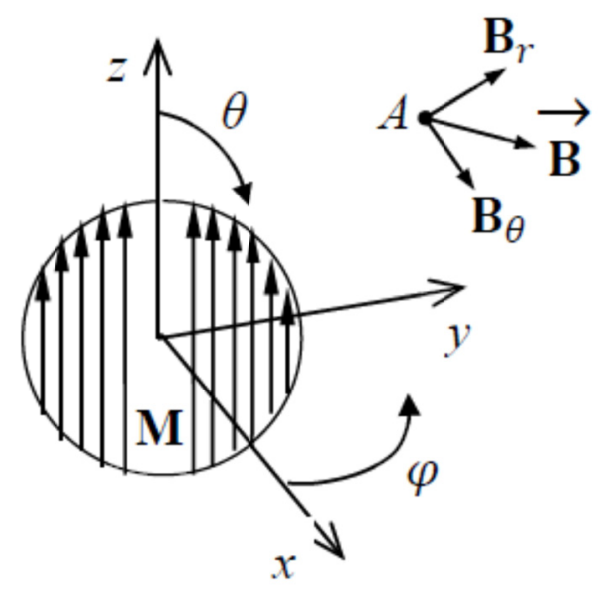

Figure 2. The uniformly magnetized sphere

For the experiment we used a $N d F e B$ sphere of diameter $d=25.4 \mathrm{~mm}$, with a magnetic moment value $p_{m} \cong 6.0 \mathrm{~A} \cdot \mathrm{m}^{2}$. A vertically positioned perforated plastic tube 6 meters long was used to guide the trajectory (Figure 3). The tube was perforated with small holes along its length to reduce aerodynamic resistance. The magnetic sphere is housed within the wooden-plastic projectile for stability during freefall and to provide the required orientation. It was dropped and allowed to free fall within the guiding tube, reaching a velocity of $v \cong 10.5 \mathrm{~m} / \mathrm{sec}$, through a circular conducting loop of radius $a$ so that $\mathbf{p}_{m}$ remained parallel to the velocity. The loop was connected to a storage oscilloscope (Tekronix TDS 210). 


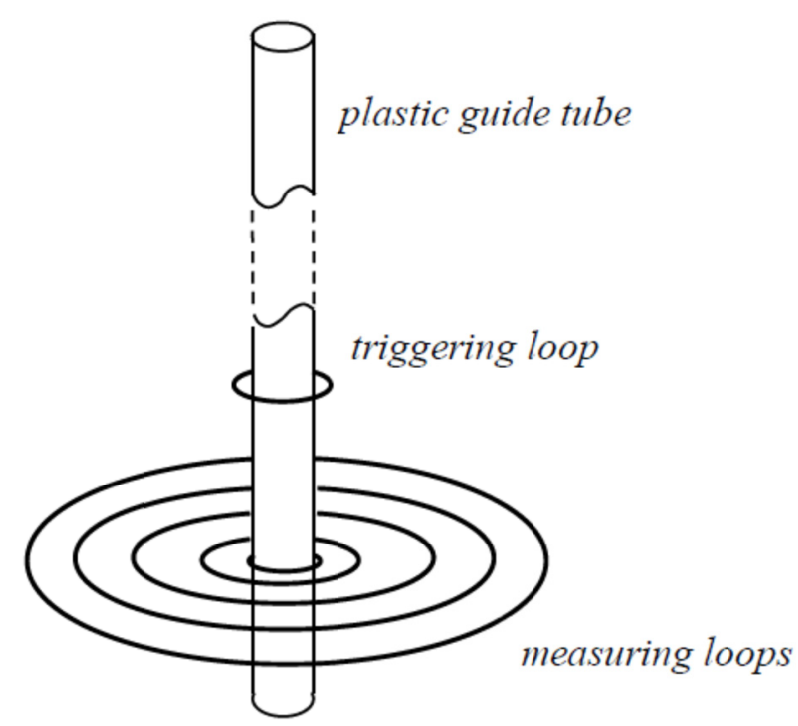

Figure 3. The free fall apparatus

It is evident that the signal from the passing sphere is dependent upon the loop's shape. Starting from Equations (1) for a circular loop with radius $a$ it is possible to derive (Taylor \& Leus, 2012) the following analytical expression for the emf induced in the loop due to Z-D effect:

$$
\operatorname{emf}(z)=\frac{3 p \pi a^{2} v z}{\left(a^{2}+z^{2}\right)^{5 / 2}} .
$$

Here $z$ denotes the position of the magnetized sphere relative to the loop centre. The corresponding signal in time has a shape shown in Figure 4 for one turn loop of radius $a=2.1 \mathrm{~cm}$. In Figure 5 we present a two-channel trace obtained for two different loops (control and testing loops) at a distance of $10 \mathrm{~cm}$ one from another. The testing loop, situated beneath the control one, is made with a thin enamelled wire wound into a spiral of $2 \mathrm{~mm}$ in diameter. As it is obvious from the photograph, both signals (left - from the control loop, right - from the testing one) are nearly identical. The conductive circular path only is essential because of the closeness of the contacts between the windings: the mutual capacitance in each coupling presents only a negligible resistance for alternating current.

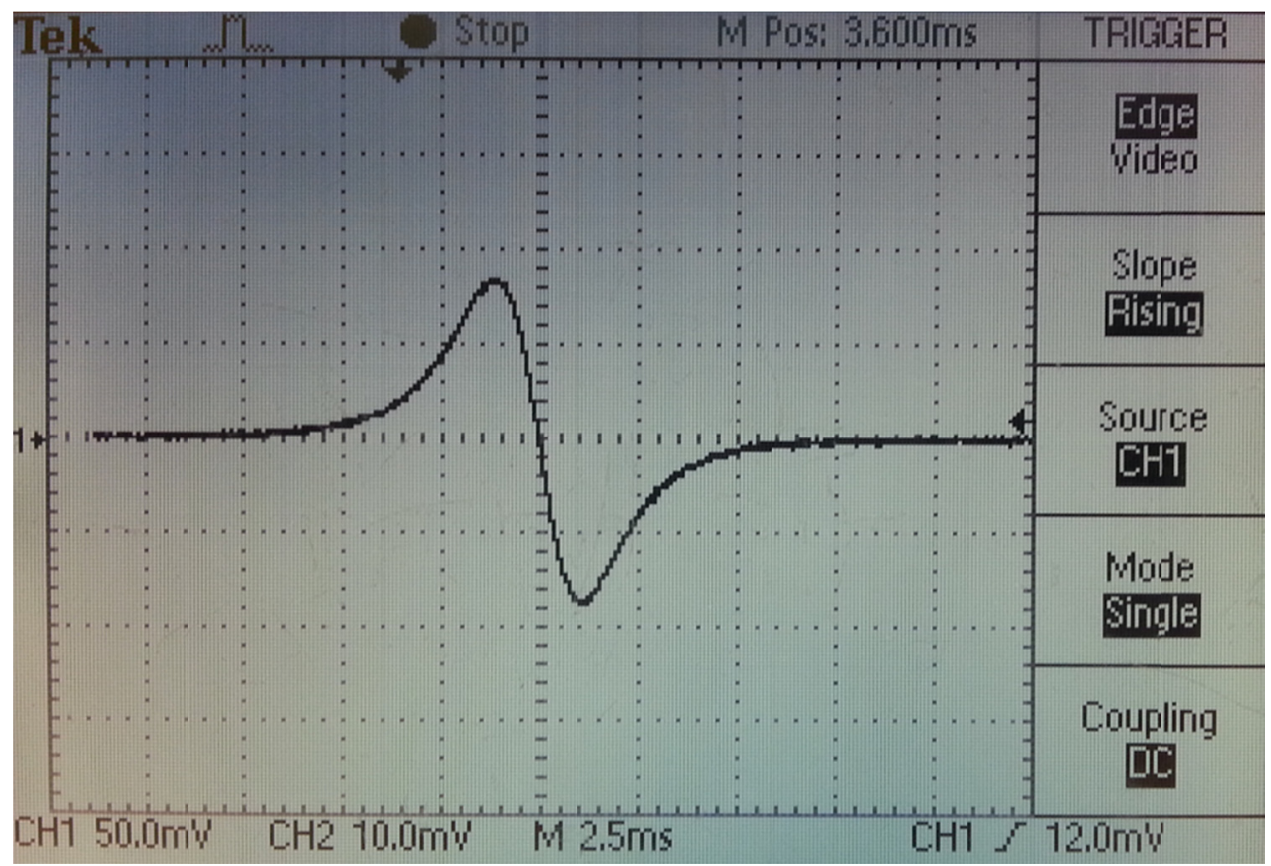

Figure 4. The signal originated from a magnetic dipole moving through circular loop 


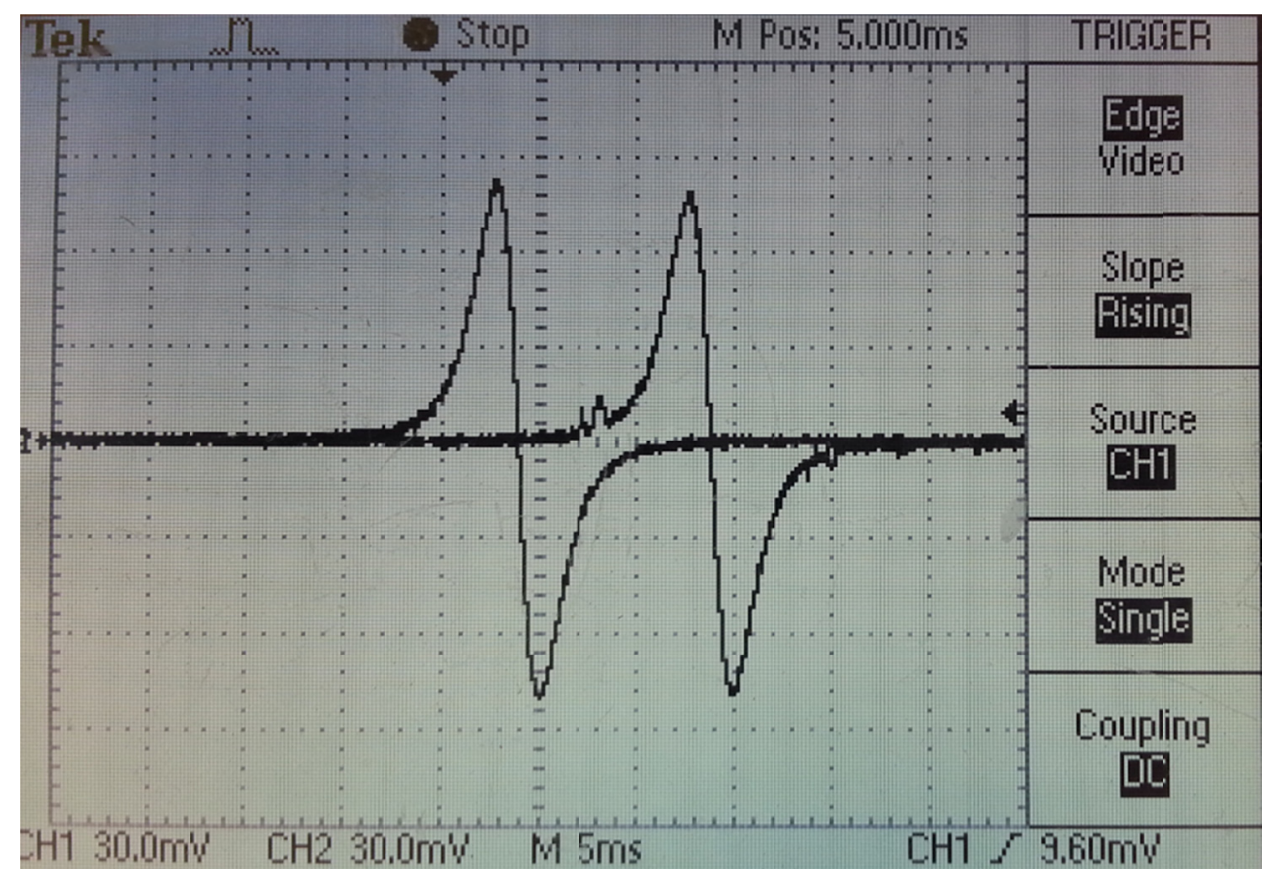

Figure 5. The signals obtained in the smooth (left) and spiralled (right) loops

To be certain, we added two equal capacitors, one on each of the leads connecting the (not spiralled) testing loop with oscilloscope. The results are presented in Figures 6 and 7. It is clear that the voltage induced in the testing loop tends to the upper limit, i.e. emf induced in the control loop, when the size of the capacitance grows.

All the details of the free fall laboratory equipment (construction of a shock absorber include) are depicted in (Taylor \& Leus, 2012). Each turn of wire in the loop increases the obtained amplitude of the signal. Two turns were used in our experimentation for the sake of doubling the value.

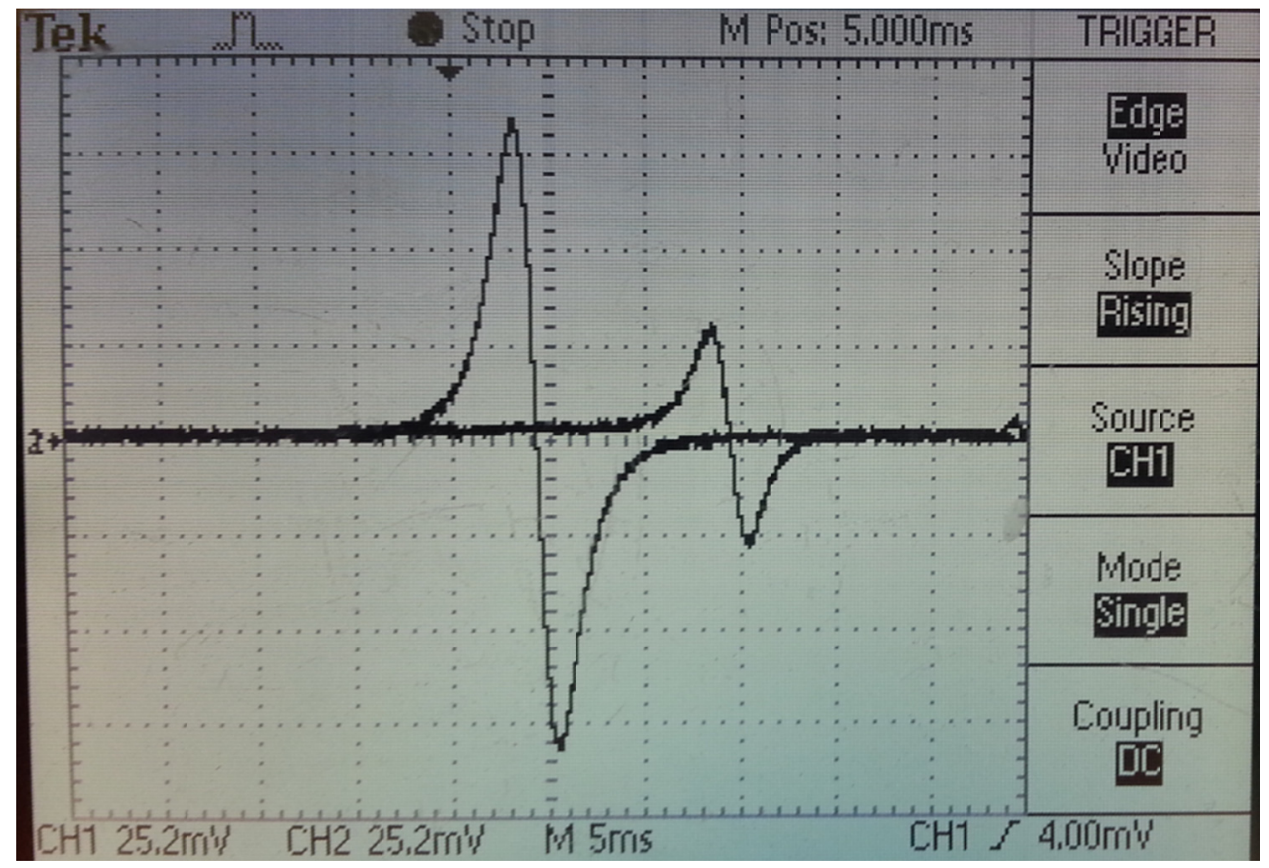

Figure 6. The signals in the control and testing loops at the capacitance $0.1 \mu \mathrm{F}$ 


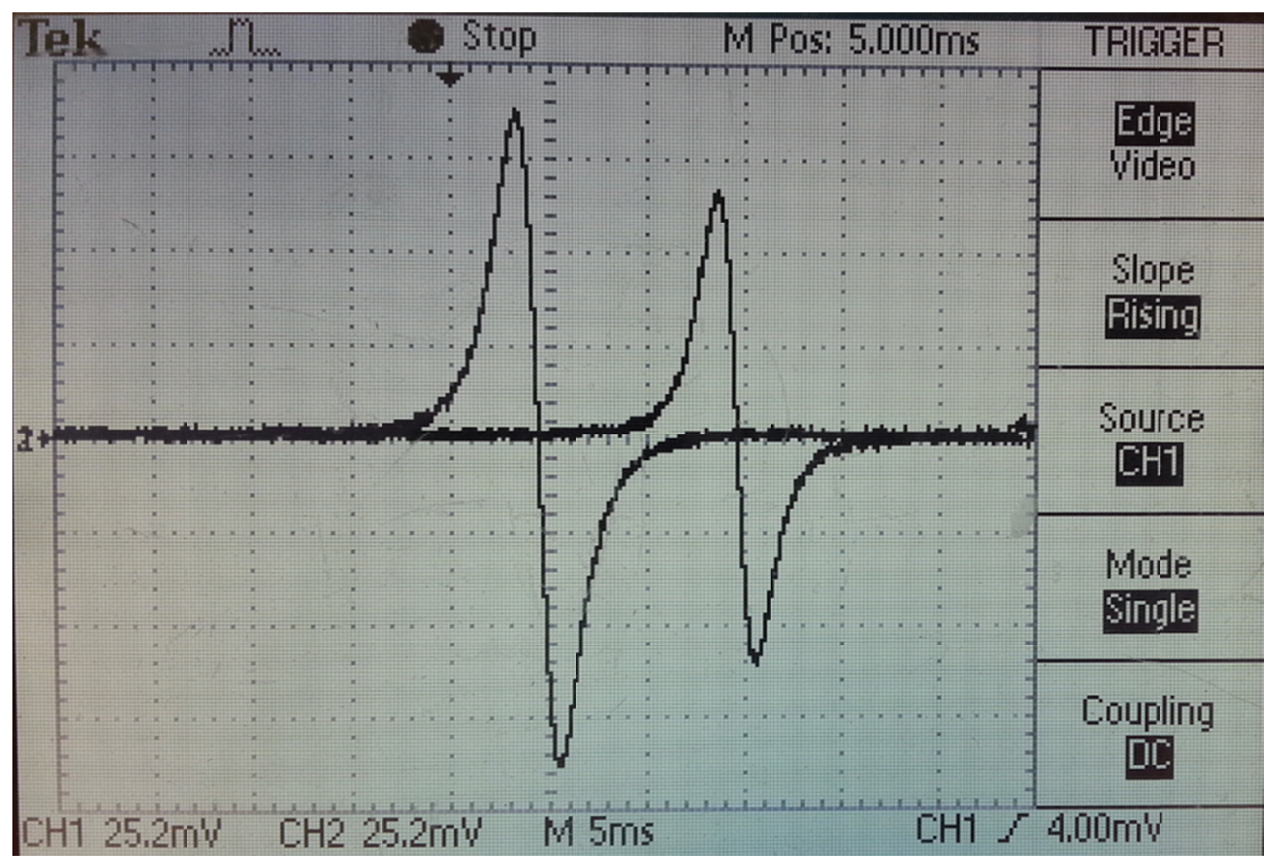

Figure 7. The signals in the control and testing loops at the capacitance $2.2 \mu \mathrm{F}$

\section{Experimental Procedure}

\section{Experiment 1.}

The above mentioned magnetised sphere was dropped and allowed to free fall down the plastic tube. At a distance of $25 \mathrm{~cm}$ from the bottom of the tube a cardboard plate with five conducting loops attached was placed horizontally (Figure 3 ). The conducting wires were arranged in concentric circles gradually increasing the radius of the loop so that the distance from the magnet will be greater in each ascending loop. The radii from the centre were approximately $3 \mathrm{~cm}, 5 \mathrm{~cm}, 7 \mathrm{~cm}, 9 \mathrm{~cm}$ and $13.5 \mathrm{~cm}$. The conducting wires were connected to a Tektronix TDS210 digital oscilloscope via a Tenma $50 \mathrm{MHz}$ oscilloscope probe. The emf generated in the each of conducting loops as the magnetic sphere passed through it gave rise to the trace seen on the screen. The resultant amplitude in $\mathrm{mV}$ was then determined and plotted on a graph against the distance from the axis.

(a)

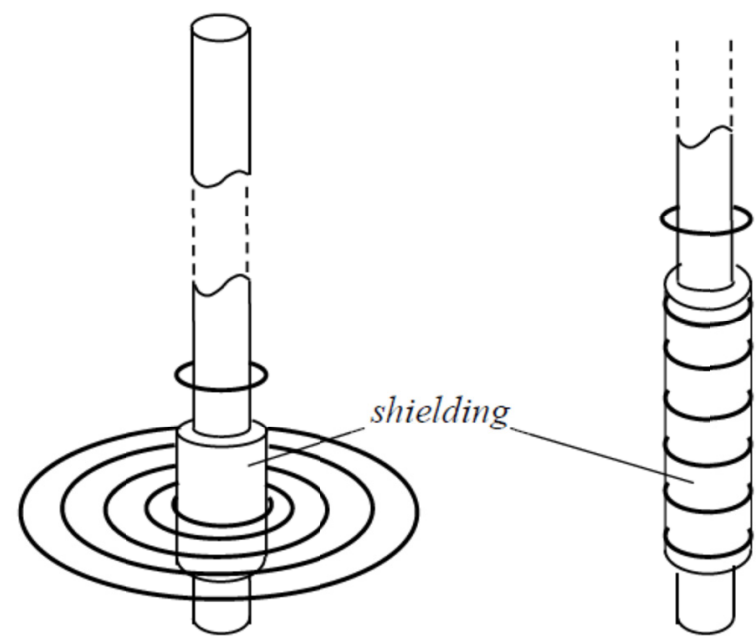

Figure 8. The free fall apparatus with shielding tube (b)

\section{Experiment 2.}

For the second experiment a soft iron tube was used to shield the conducting loops from the magnetised sphere passing through them (Figure 8a). We used two different soft iron tubes to shield a segment of the magnetized 
sphere trajectory:

1. $5 \mathrm{~cm}$ in diameter, $4 \mathrm{~mm}$ thickness of the wall, and $23 \mathrm{~cm}$ long

2. $6 \mathrm{~cm}$ in diameter, $4 \mathrm{~mm}$ thickness of the wall, and $23 \mathrm{~cm}$ long

In both cases the shielding tube was placed at the bottom of the guiding tube and the cardboard plate was positioned at $1 \mathrm{~cm}$ from the upper brim. The smallest diameter loop had to be omitted from this experiment due to the width of the iron shielding.

\section{Experiment 3.}

For the third experiment the cardboard plate was removed and a conducting loop of diameter compatible with that of the shielding tube was placed at the top of the shielded tube and moved vertically down the tube at some intervals as shown in Figure 8b. The result of the passing magnetised sphere was recorded before moving on to the next interval and repeating the free fall experiment.

\section{Experimental Results}

The above mentioned magnetized sphere induces an emf inside a circular loop when passing through it. The theoretical form of the signal is given by Equation (2), which defines the two extremities. The extremity values are provided by the formula

$$
E_{v}^{\max }= \pm 2.7 \frac{p v}{a^{2}}
$$

where the value $p$ may be expressed through the magnetic moment of the sphere (Taylor \& Leus, 2012):

$$
p=\frac{\mu \mu_{0} p_{m}}{2 \pi}=1.21 \times 10^{-6} .
$$

The positions of two extreme values of emf are given by formula

$$
z_{1,2}= \pm \frac{a}{2}
$$

In the following text the word "amplitude" means the peak-to-peak variation. Figure 9 shows:

i. The dependence of amplitude on the radial distance $a$, calculated according to the Formula (3);

ii. The dependence of amplitude on the radial distance $a$, measured for the six gradually increasing values (experiment 1);

iii. The dependence of amplitude on the radial distance $a$, measured for the five values when using the soft iron shielding (experiment 2).

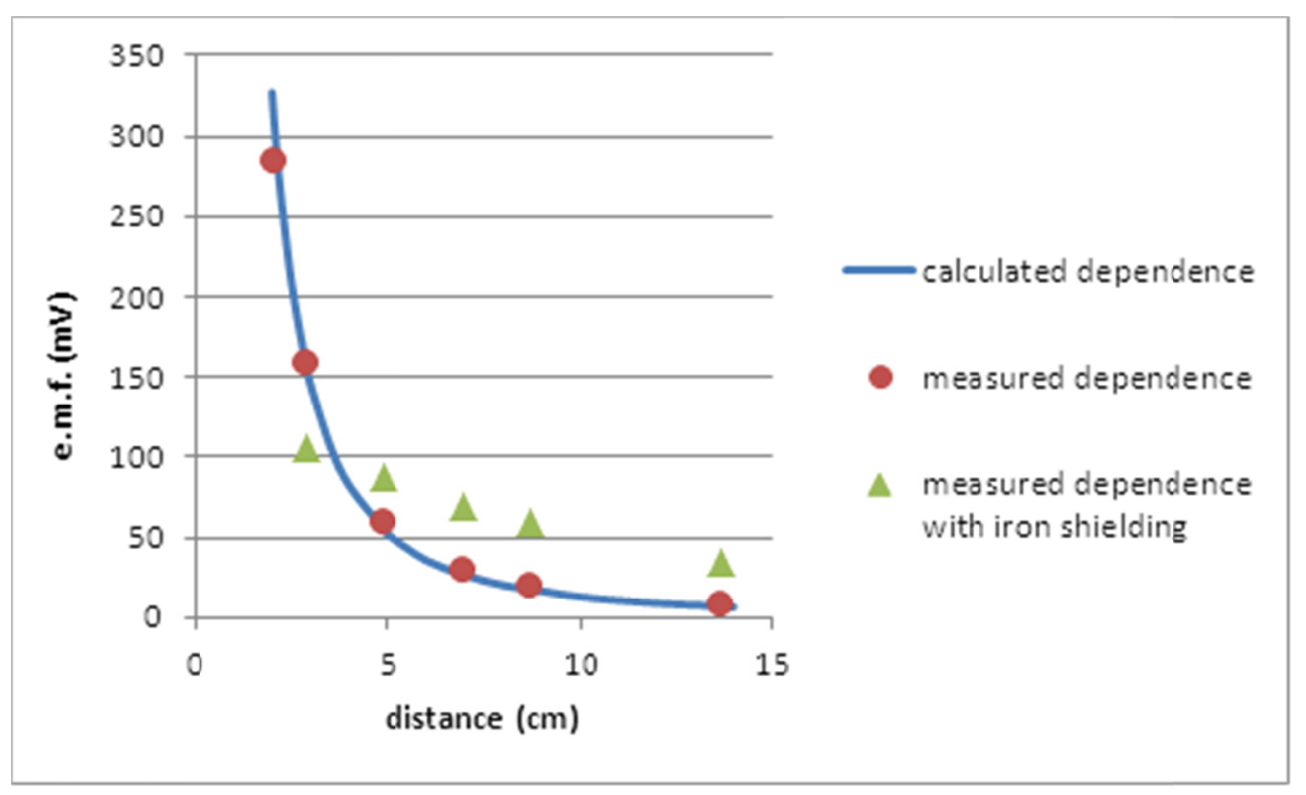

Figure 9. Induced emf signal for different distances from the axis in horizontal plane 


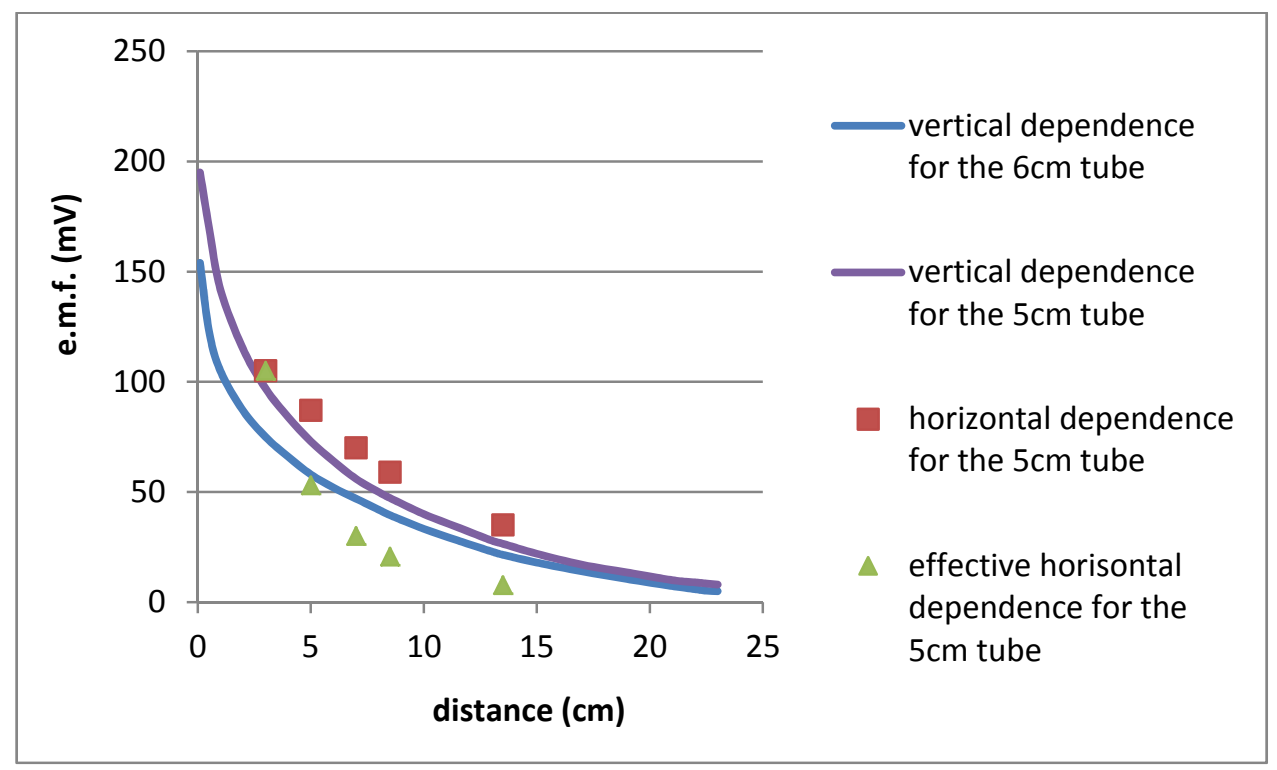

Figure 10. Induced emf signal for iron shielding with the measuring loop located at different distances

Figure 10 shows the result of the amplitude measurement for twenty two points of vertical displacement of the measuring loop relative to the top brim of the shielding tube (experiment 3). These discrete data sets are presented with smoothed lines. In addition, the previous data is presented in the discrete form. In total we have:

i. Line obtained with $5 \mathrm{~cm}$ tube shielding,

ii. Line obtained with $6 \mathrm{~cm}$ tube shielding.

iii. The horizontal dependence with $5 \mathrm{~cm}$ tube shielding (experiment 2).

iv. The horizontal dependence with $5 \mathrm{~cm}$ tube shielding divided by the length of the loop. This ratio, accounting for an increase in the source of the emf, gives due parity for both vertical and horizontal dependencies.

\section{A Patent Separation Between Electro-Kinematical and Electro-Dynamical Signals}

A further experiment was devised and carried out. Instead of a direct connection between the measuring loop and an oscilloscope a different method of detecting the passing magnetic signal is used and outlined below (Figure 11). The amplitude of a pulse induced in a circular loop is proportional to the inverse square of its radius (Equation 3). Therefore the electro-kinematic power decreases much more rapidly when compared to the electro-dynamic power as the distance between the measuring conductor (circular antenna) and the source of pulse (metal tube which the magnetic sphere pass through) increases (Figure 9). The maximum conducing circle we used was of $65 \mathrm{~cm}$ in diameter. At such a distance from the guide-tube the signal we are trying to observe becomes indistinguishable from the electromagnetic noise in the laboratory. For this reason we have been compelled to resort to additional circuitry before passing the signal to the oscilloscope, based on the method of active frequency filtration.

A polygonal antenna, consisting of a single turn of wire, was constructed and placed around the guide tube. A Low Pass Filter was installed in series with the antenna that rejected all high frequency interference. We established a corner frequency of $1 \mathrm{kHz}$ in order to allow passage of our signal (fundamental frequency of $500 \mathrm{~Hz}$ ) but reject any small amplitude signals at frequencies above this.

An inverting amplifier circuit was used with a gain set to approximately 1000 , allows a good enough resolution over the output range $-9 \mathrm{~V}$ to $+9 \mathrm{~V}$ to be able to detect very small signals of the kind we struggled to observe in previous experiments. 


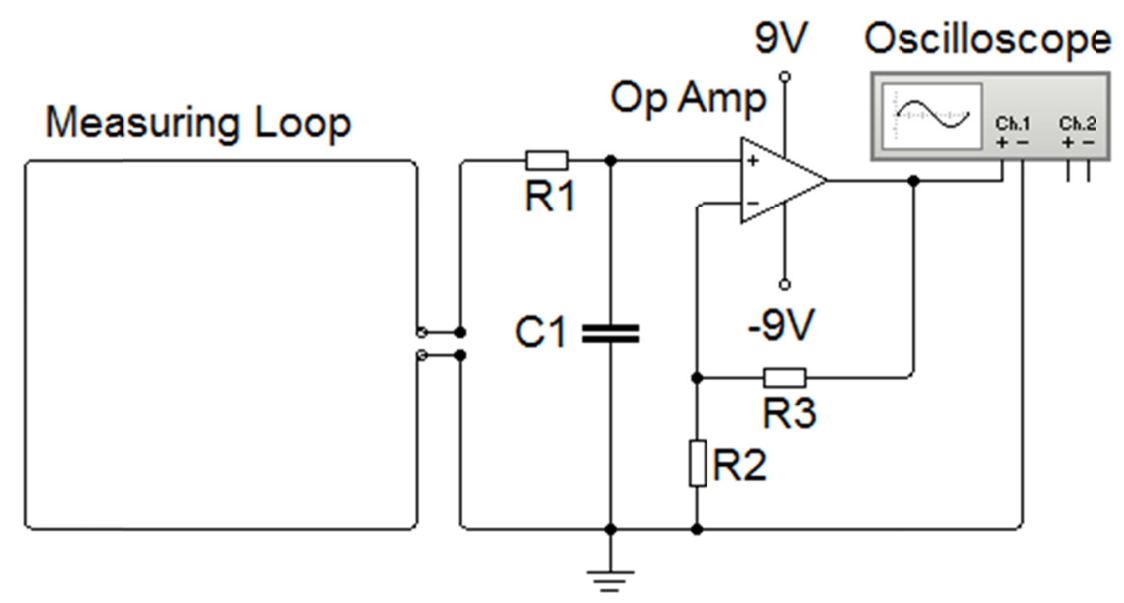

Figure 11. Scheme of the signals separation

\section{Commentary of the Results and Conclusion}

The main quality of the experiments carried out is to be able to appreciate causes through a reciprocal comparison of their effects. As it may be seen in Figure 9, the magneto-kinematical emf (whole curve) has a very dissimilar special distribution with regard to the distribution of the emf obtained under shielding condition. A kinematically induced electric field $\mathbf{E}=\mathbf{B} \times \mathbf{v}$, measured in the laboratory through the apparent emf , is almost entirely cancelled everywhere outside of the shielded iron tube. Let us remark that in the latter case the source of emf is always situated at the brim (input and output) of the shielding tube. Both arising pulses (Figure 12) are separated by twenty five milliseconds - time interval corresponding to the distance of $25 \mathrm{~cm}$ at the speed $v=10 \mathrm{~m} / \mathrm{sec}$. And what event occurs there? As soon as the magnetized sphere comes close to the input brim of the shielding tube, its magnetic field undergoes a complicated intrinsic transformation. Instead of being spread out over space it curls up into the narrow confinement of the tube wall. Therefore, we have all conditions necessary for Faraday's law to be manifested:

$$
\boldsymbol{\nabla} \times \mathbf{E}=-\frac{\partial \mathbf{B}}{\partial t} .
$$

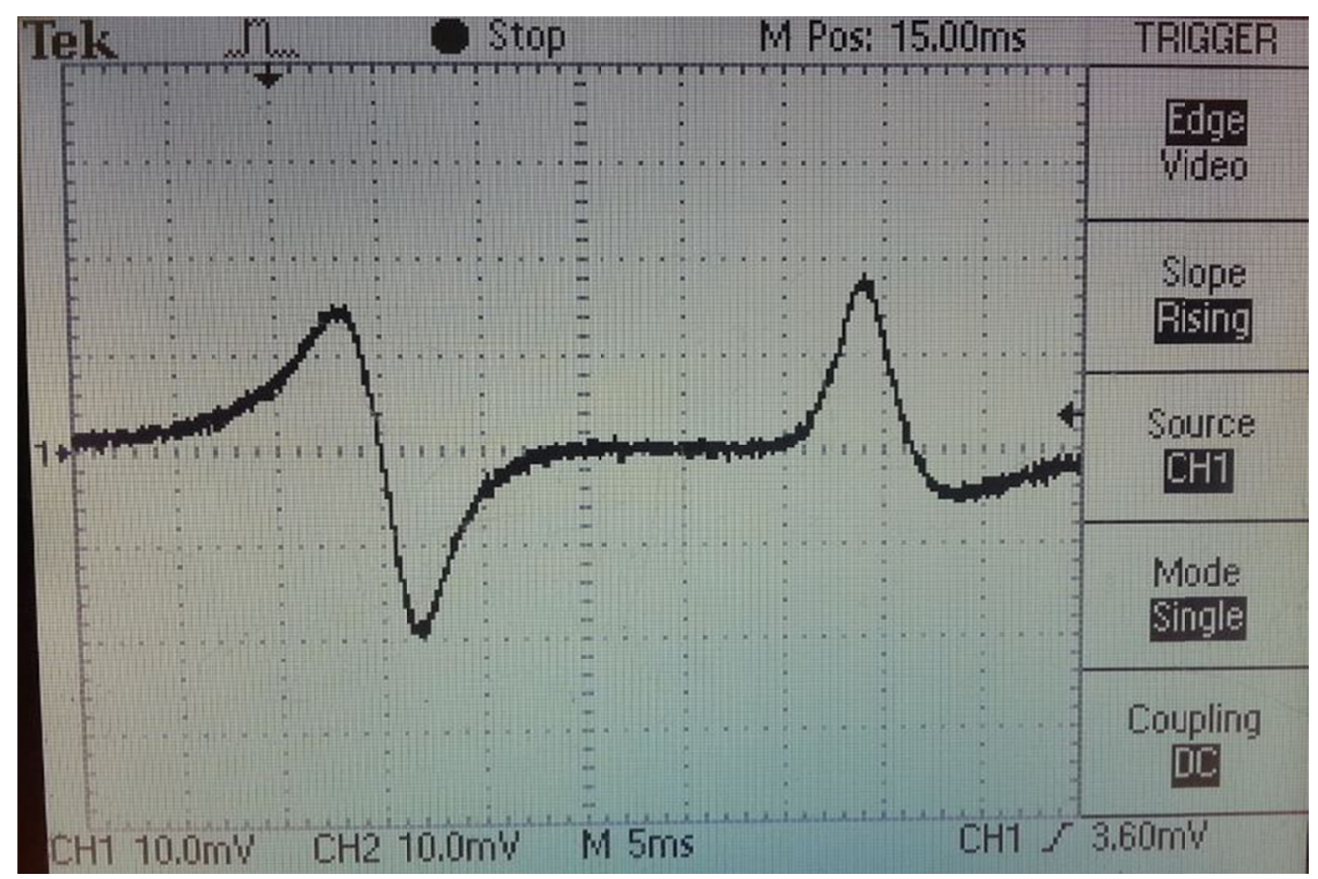

Figure 12. Magneto-dynamic pulses induced in the measuring loop shielded with iron tube 
Z-D effect appears as the emf induced due to a moving magnetic field. In contrast to Z-D effect, Faraday's law manifests itself in the emf provided by a magnetic field explicitly varying over time. A good illustration for that is the result of vertical measurement with the iron tube of $5 \mathrm{~cm}$ in diameter. The correspondent amplitudes are just $20 \%=(6-5) / 5$ bigger than amplitudes in the case of the $6 \mathrm{~cm}$ tube (Figure 10). It would be understandable if we have to regard the following essential peculiarity. With a narrower tube the magnetic field of the passing sphere is more compressed over the same time, so the derivative $\partial \mathbf{B} / \partial t$ inevitably augments.

Given that this entity is inferred, we could expect a similar effect for a paramagnetic material. Paramagnetism is like ferromagnetism "in miniature", therefore an experimental check is more difficult to perform but yet feasible. An aluminium cylinder of $5 \mathrm{~cm}$ in diameter, $4 \mathrm{~mm}$ thickness of the wall, and $23 \mathrm{~cm}$ long was fixed on the guide-tube with a measuring loop located at the centre so that both brims are equi-distant from the circular loop producing emf. The resulting signal for a circular loop of $14 \mathrm{~cm}$ in diameter is presented in Figure 13. The shielding effect is rather moderate but two distinct signals can be observed developing at the top (first) and the bottom (second) brims. A time interval of approximately twenty five milliseconds between the two signals can also be deduced from the oscilloscope trace. A paramagnetic material is almost transparent for the permanent magnetic field that is why in the case of aluminium shielding we observe a self style superposition picture of both - magneto-kinematic and magneto-dynamic effects in contrast to the ferromagnetic shielding (Figure 12).

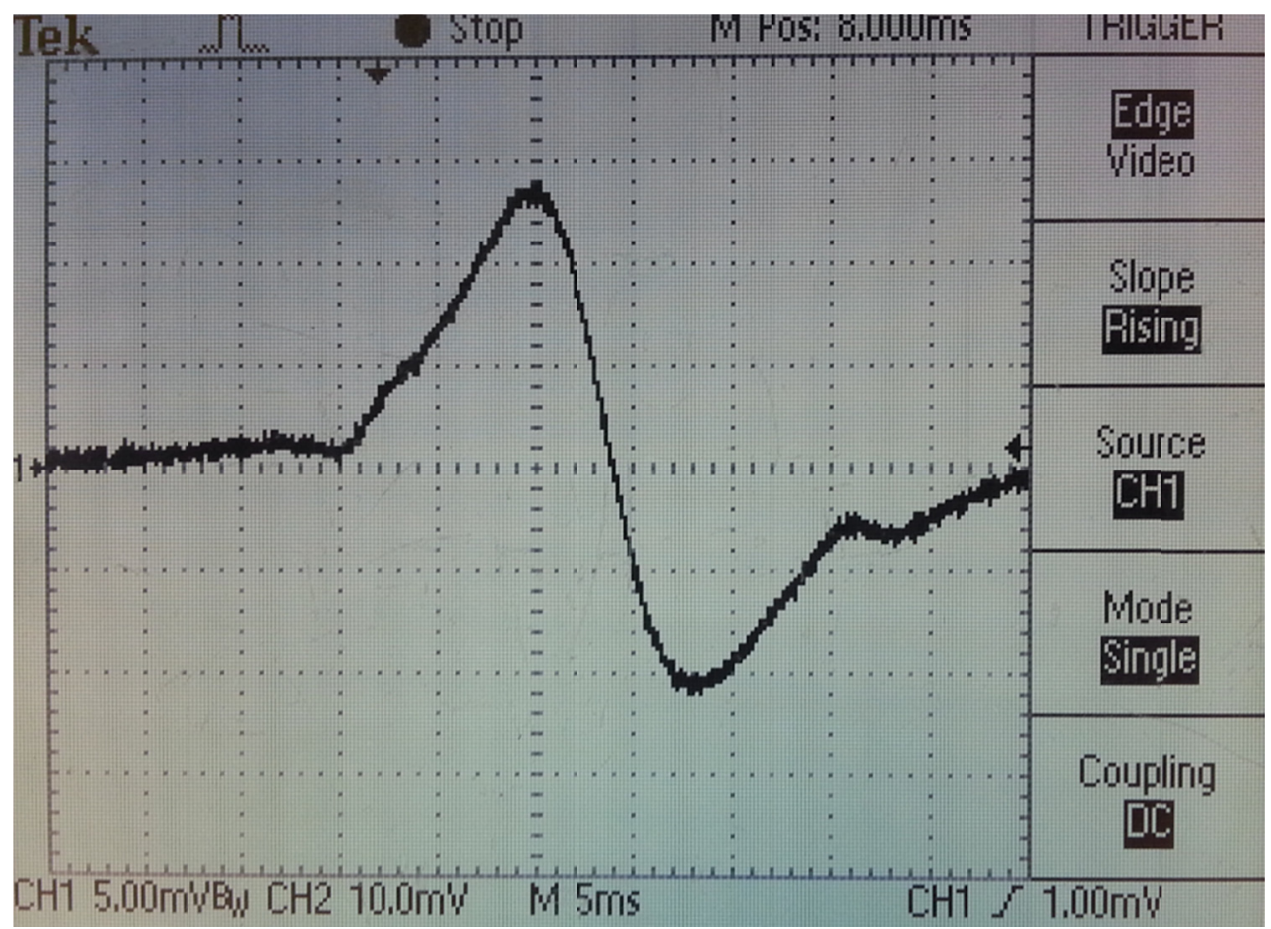

Figure 13. Superposition picture of magneto-kinematic and magneto-dynamic effects

For the iron tube $37 \mathrm{~cm}$ long the signal from circular antenna of $65 \mathrm{~cm}$ in diameter is present on Figure 14 . Two purely electro-dynamic pulses - from input rim and output rim - have identical form with amplitudes equal to almost $5 \mathrm{~V}$ as a consequence of amplification in the filter device. The corresponding electro-kinematic pulse is entirely absorbed in the ferromagnetic material, so no superposition is observed unlike the case of aluminium.

In accordance with (4), the time interval between minimal and maximal voltage in an electro-kinematic pulse is $\Delta t=a / v$, which gives for $a=7 \mathrm{~cm}$ and $v=10 \mathrm{~m} / \mathrm{sec}$ the value $\Delta t=7 \mathrm{~ms}$. So we see a superposed mixture of both electro-kinematic and electro-dynamic pulses in the case of the aluminium tube (Figure 13). For our $65 \mathrm{~cm}$ antenna $\Delta t=32.5 \mathrm{~ms}$, while the time interval between input-originated pulse and outputoriginated pulse is $\cong 23 \mathrm{~ms}$ because the aluminium tube is $23 \mathrm{~cm}$ long. The small and widespread electro-kinematic pulse therefore is indiscernible against two electro-dynamic pulses (Figure 15), although their amplitude is five times smaller than in the case of iron tube. 


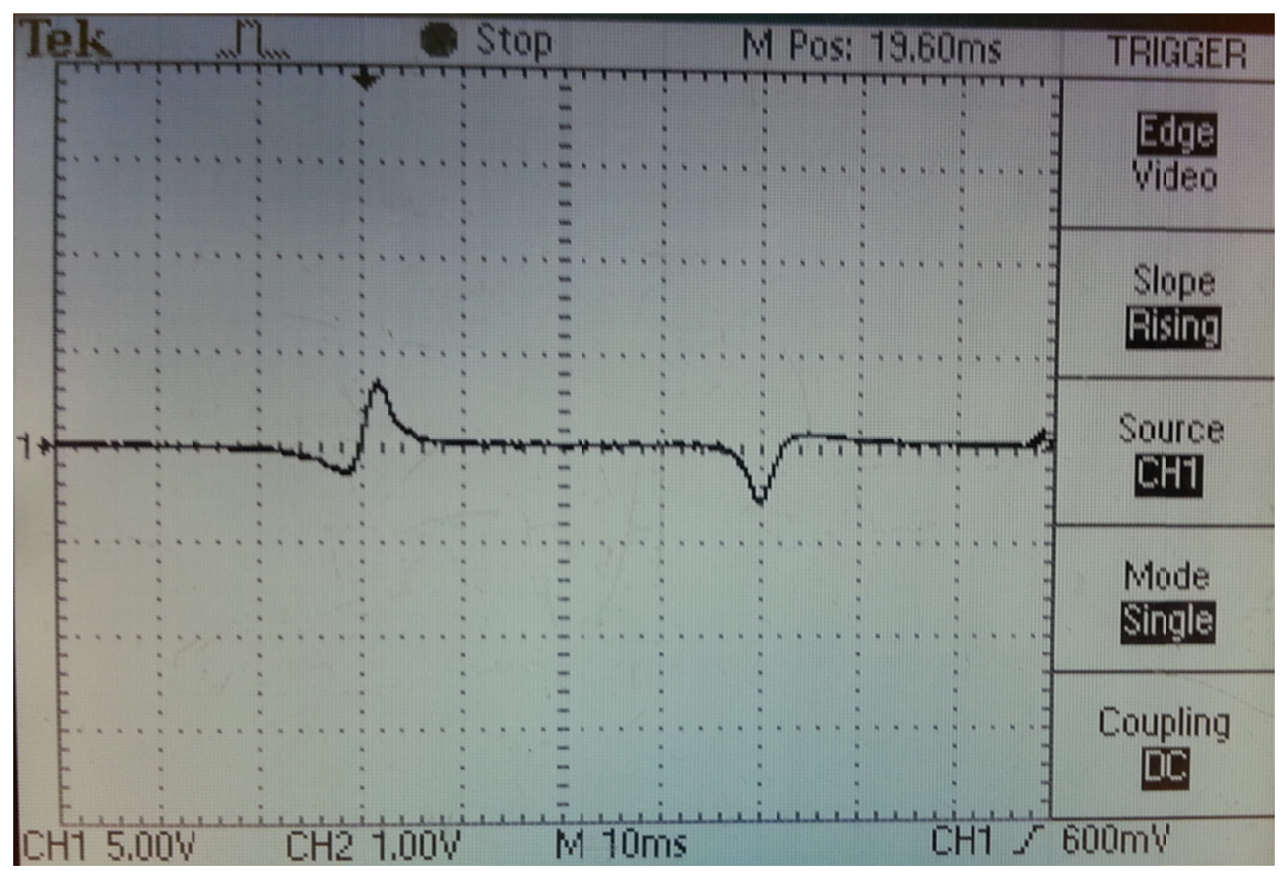

Figure 14. Antenna signal for the iron tube as a source

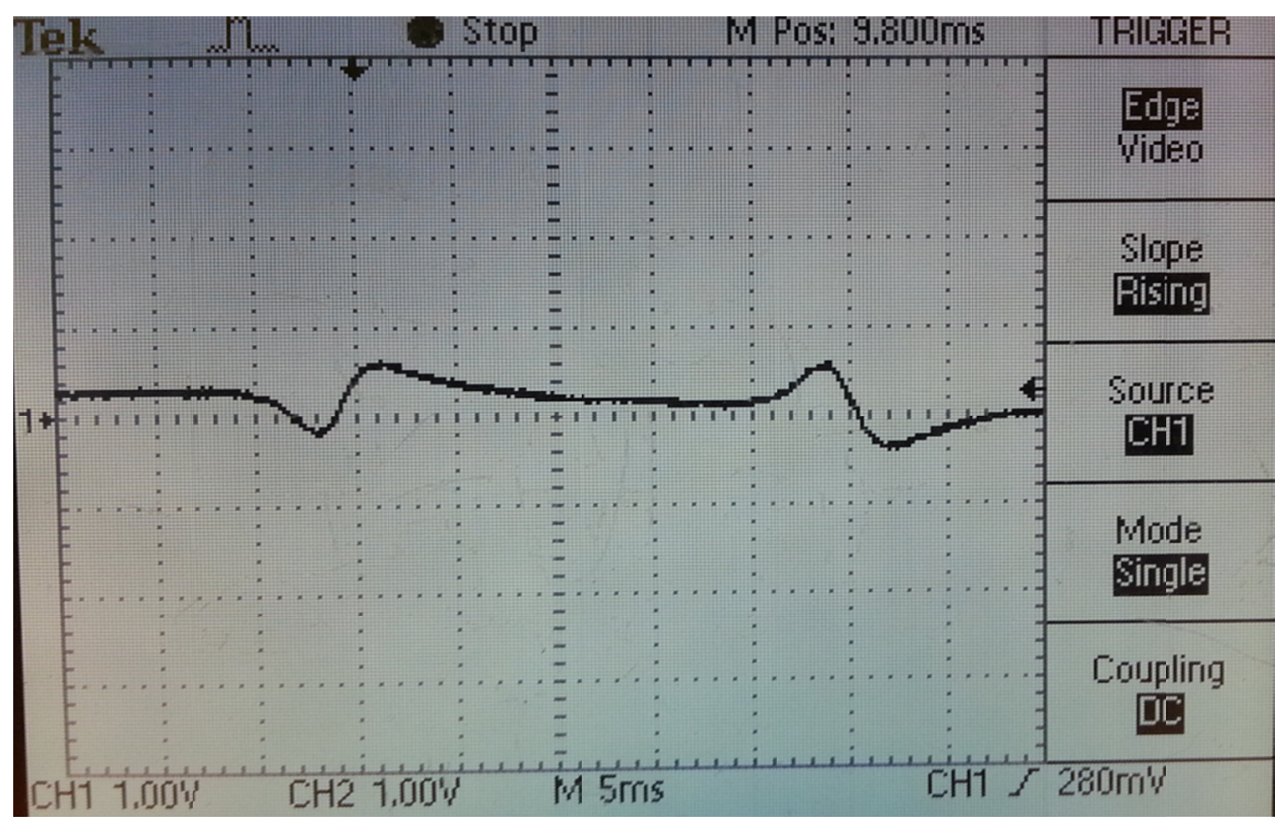

Figure 15. Antenna signal for the aluminium tube as a source

We do not dispose of a circular frame bigger than $65 \mathrm{~cm}$ in diameter, and so the further measurements were carried out with a stretched wire circumscribing a polygon among some furniture items. The first polygon had $310 \mathrm{~cm}$ in the average diameter, and we have obtained a distinct signal originated from the iron tube $37 \mathrm{~cm}$ long and $5 \mathrm{~cm}$ in diameter (Figure 16). As it can be seen, the noise level gets comparable with the useful signal amplitude. 


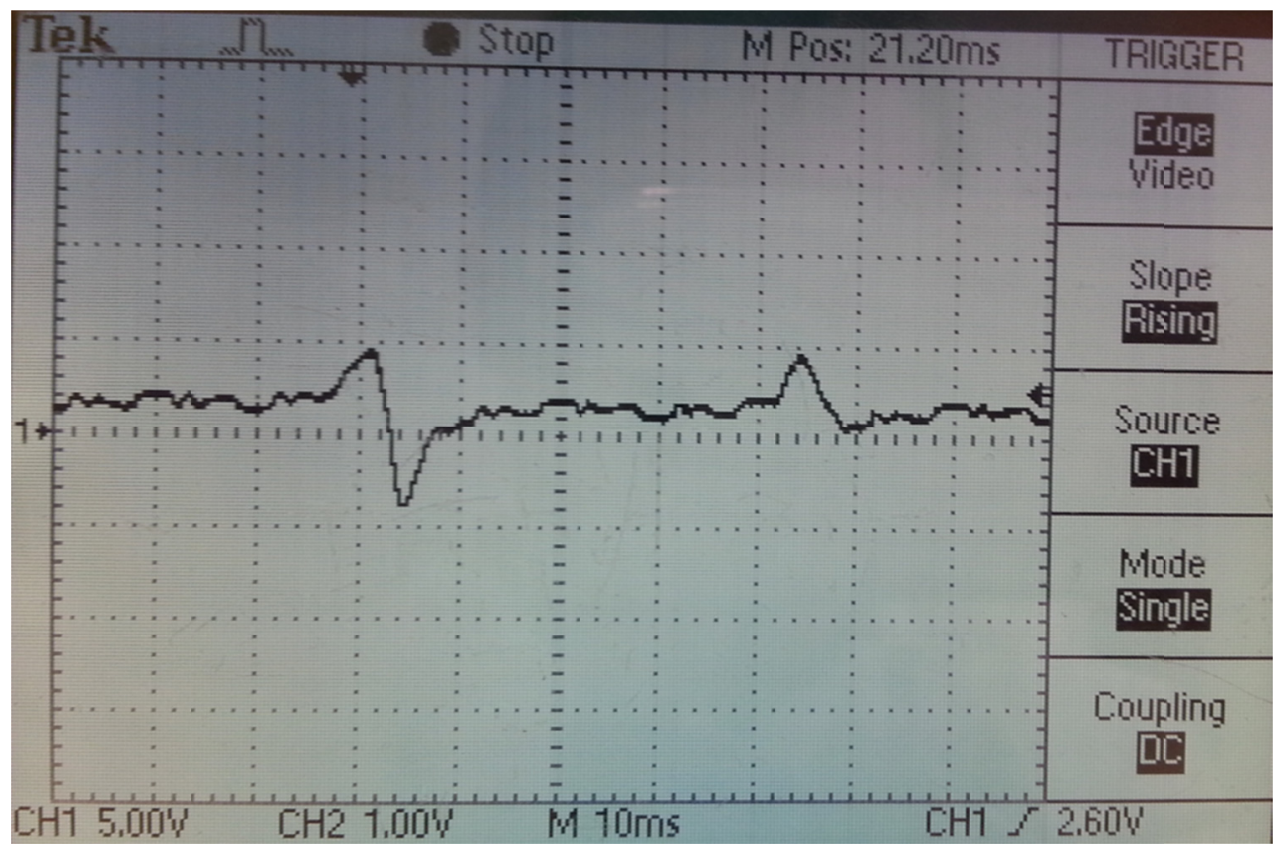

Figure 16. Polygonal antenna signal for the iron tube as a source

The second polygon had $230 \mathrm{~cm}$ in average diameter where a weak signal from aluminium tube $23 \mathrm{~cm}$ long had been observed (Figure 17). It is at the limit of our ability to separate the interested signal relative to the noise level with the current amplifying instrument. Due to the Formula (4) the duration of electro-kinematical signal would be bigger than $115 \mathrm{~ms}$. Not only is this value much bigger than $23 \mathrm{~ms}$ but our shock-absorber is situated nearer than $30 \mathrm{~cm}$ from the output rim of the aluminium tube. So the influence of the electro-kinematic pulse is thoroughly ruled out. These are two absolutely different natural phenomena, and it is the notorious "flux rule" that is steadily blurring the line between kinematic and dynamic types of induction (Leus \& Taylor, 2011).

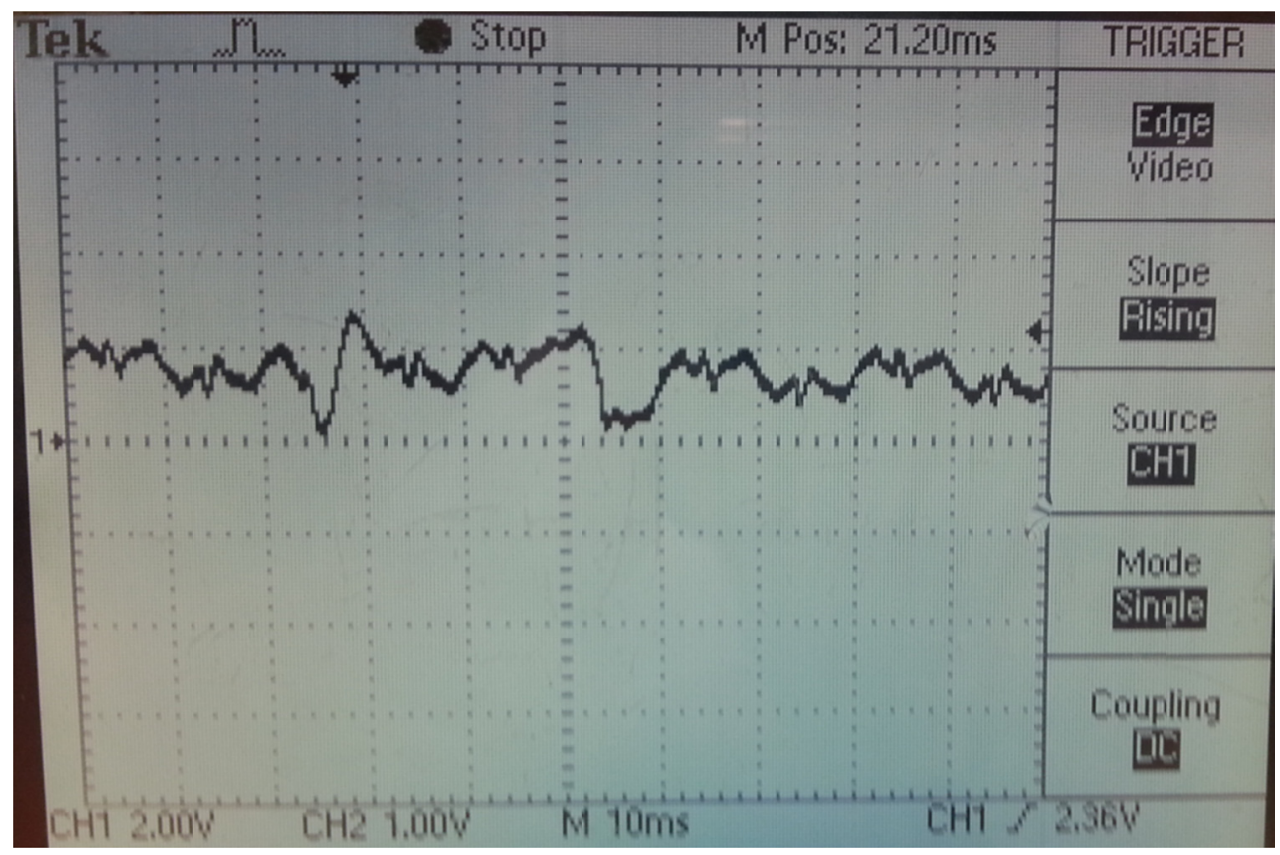

Figure 17. Polygonal antenna signal for the aluminium tube as a source 
A shielding tube, surely, may be represented as a series of conducting rings and, as soon as the magnetized sphere is falling under the influence of gravity, eddy currents are circulating inside them. However, their contribution is not appreciable as it is evident from the obtained photographs: we have only two separated pulses without any trace of signal between. The responsibility for causing magneto-electric pulses in the effect under consideration rests with the microscopic circular currents which are a source of the macroscopic magnetic field (Ampere). When the magnetized sphere enters the shielding tube all Ampere's currents in the metal of the tube change their spatial orientation. So the very source of the electromagnetic radiation in this respect is supposedly the body of the metal screen surrounding the passing magnetized sphere.

In conclusion, we would wish to express a hope that our finding will serve not only for declaring the evidence of a new source of electromagnetic radiation but also as an instrument for studying inherent structure of magnetic materials.

\section{References}

Eihenwald, A. A. (1956). Izbrannye raboty - Selected works (Moscow: Physico-matematicheskaja literature, in Russian).

Jackson, J. D. (1998). Classical Electrodynamics (3rd ed.). New York: John Wiley.

Kelly, A. G. (2004). Unipolar Experiments. Annales de la Foundation Luis de Broglie, 29(1-2), 119-148.

Leus, V., \& Taylor, S. (2011). On the motion of the field of a permanent magnet. Eur. J. Phys., 32(5), 1179-1192. http://dx.doi.org/10.1088/0143-0807/32/5/006

Leus, V. A. (2013). Magneto-kinematical and Electro-kinematical Fields. Progress in Electromagnetics Research-M, 32, 27-41. Retrieved from http://www.jpier.org/PIERM/pierm32/03.13043008.pdf

Leus, V. A., \& Zatolokin, V. N. (2006). The magnetokinematical effect. IJEEE, 43(4), 245-251.

Rowland, H. A. (1878). On the magnetic effect of electric convection. American Journal of Science, 15, 30-38. http://dx.doi.org/10.2475/ajs.s3-15.85.30

Taylor, S., \& Leus, V. (2012). The magneto-kinematic effect for the case of rectilinear motion. Eur. J. Phys., 33(4), 837-852. http://dx.doi.org/10.1088/0143-0807/33/4/837

Zajev, N. E., \& Dokuchajev, V. I. (1964). About the behaviour of force lines of the field of a rotating magnet. Electrotechnika (Electrical engineering, in Russian), 11, 64.

\section{Copyrights}

Copyright for this article is retained by the author(s), with first publication rights granted to the journal.

This is an open-access article distributed under the terms and conditions of the Creative Commons Attribution license (http://creativecommons.org/licenses/by/3.0/). 